\title{
BMJ
}

\section{Antipsychotic drugs and risk of venous thromboembolism: nested case-control study}

\author{
Chris Parker, medical statistician, ${ }^{1}$ Carol Coupland, associate professor in medical statistics, ${ }^{2}$ Julia Hippisley- \\ Cox, professor of clinical epidemiology and general practice ${ }^{2}$
}

${ }^{1}$ Nottinghamshire County Teaching Primary Care Trust, Hucknall Health Centre, Hucknall, Nottingham NG15 7JE

Division of Primary Care, University Park, Nottingham NG2 7RD

Correspondence to:J Hippisley-Cox juliahippisleycox@gmail.com

Cite this as: BMJ 2010;341:C4245 doi:10.1136/bmi.c4245

\section{ABSTRACT}

Objective To determine whether antipsychotic drugs are associated with an increased risk of venous thromboembolism, and to examine risks by type of antipsychotic, potency, and dose.

Design Population based nested case-control study. Setting The UK QResearch primary care database. Participants Patients (cases) with a first ever record of venous thromboembolism between 1 January 1996 and 1 July 2007; each was matched with up to four controls by age, calendar time, sex, and practice.

Main outcome measures Odds ratios for venous thromboembolism associated with antipsychotic drugs adjusted for comorbidity; concomitant drug exposure. Results There were 25532 eligible cases (15975 with deep vein thrombosis and 9557 with pulmonary embolism) and 89491 matched controls from a study population of 7267673 . Individuals prescribed antipsychotic drugs in the previous 24 months had a $32 \%$ greater risk of venous thromboembolism than non-users, despite adjustment for potential risk factors (odds ratio $1.32,95 \%$ confidence interval 1.23 to 1.42 ). Patients who had started a new drug in the previous three months had about twice the risk $(1.97,1.66$ to 2.33$)$. The risk was greater for individuals prescribed atypical rather than conventional drugs (adjusted odds ratio 1.73, 1.37 to 2.17 , for atypical drugs; $1.28,1.18$ to 1.38 , for conventional drugs). It also tended to be greater for patients prescribed low rather than high potency drugs $(1.99,1.52$ to 2.62 , for low potency; $1.28,1.18$ to 1.38 , for high potency). The estimated number of extra cases of venous thromboembolism per 10000 patients treated over one year was 4 ( 3 to 5 ) in patients of all ages and 10 (7 to 13) for patients aged 65 and over.

Conclusions There is an association between use of antipsychotic drugs and risk of venous thromboembolism in a large primary care population. The increased risk was more marked among new users and those prescribed atypical antipsychotic drugs.

\section{INTRODUCTION}

Venous thromboembolism is an important and preventable cause of morbidity and mortality. ${ }^{1} \mathrm{Up}$ to a quarter of affected patients die within a week, ${ }^{2}$ and almost a third of survivors experience long term effects. ${ }^{34}$ Some research has indicated that antipsychotic drugs, some of which are also widely prescribed for nausea, vomiting, and vertigo, might be associated with an increased risk of venous thromboembolism. Early case reports led to several studies, but the findings have been inconsistent and the possible association has received little attention.

One case-control study found a sevenfold risk of venous thromboembolism among current users of antipsychotic drugs, ${ }^{5}$ and another a 13 -fold risk of death from pulmonary embolism. ${ }^{6}$ The studies were based on small numbers, excluded older people, and were conducted before the widespread use of atypical antipsychotic drugs. A hospital based case-control study found a 3.5fold risk associated with antipsychotics, with a lower risk for atypical drugs than for conventional drugs. ${ }^{7}$ Two large cohort studies focused on people aged 65 or over who were taking antipsychotic drugs. ${ }^{89}$ One found a nonsignificant overall association, ${ }^{8}$ although the subgroup taking butyrophenone conventional drugs showed a $43 \%$ increased risk. The other, based on residents of nursing homes, found a doubled risk associated with atypical drugs but no effect with conventional drugs. ${ }^{9}$ A further cohort study focused on the atypical clozapine and found that current users had a fivefold risk of death from pulmonary embolism compared with past users. ${ }^{10}$

There are therefore grounds for concern, but considerable uncertainty remains on an association that, if proved, would have important implications. Several plausible biological mechanisms have been suggested, including enhanced aggregation of platelets, raised concentrations of anticardiolipin antibodies, and exacerbation of venous stasis. ${ }^{11}$

We investigated whether antipsychotic drugs are associated with an increased risk of venous thromboembolism and examined risks by type, potency, and dose, adjusting for comorbidity and concomitant drug exposure. We carried out a nested case-control study in a large population based primary care cohort in the United Kingdom.

\section{METHODS}

\section{Data source}

The QResearch database version 16 (www.qresearch. org) holds the anonymised primary care clinical 
records of over 11 million people registered at any time in the past 16 years with $525 \mathrm{UK}$ general practices. The database includes data on patients' demographics, characteristics, consultations, symptoms, diagnoses, referrals, results of investigations, and prescribed drugs. It has been subject to detailed analyses including age-sex distributions, birth rates, death rates, and consultation rates and shows good correspondence with other sources. ${ }^{12}$ Recording of clinical diagnoses including psychotic illness ${ }^{13}$ and venous thromboembolism ${ }^{14}$ has good levels of completeness and accuracy in UK general practice databases.

\section{Study population}

The study population consisted of an open cohort of patients registered with participating practices between 1 January 1996 and 1 July 2007. Cases were all patients aged between 16 and 100 with a first ever record of venous thromboembolism (deep vein thrombosis or pulmonary embolism) during the study period, including postmortem diagnoses, identified from computer recorded Read codes (diagnostic codes) for venous thromboembolism recorded within the patient's electronic record.

We used incidence density sampling to identify up to four controls for each case, matched by single year of age, calendar time, sex, and practice. All controls were alive and registered with the practice at the date of the first recorded diagnosis of venous thromboembolism in their matched case: this was the index date for each case and their controls. Controls had no diagnosis of venous thromboembolism in their record up to this date.

\section{Exclusions}

Potential participants (cases and controls) were excluded if they had less than 24 months of data before the index date or if area census data on socioeconomic status were missing from their record (usually temporary residents). Controls were excluded if they had had any prescriptions for warfarin before the index date, as this could be treatment for a previous, unrecorded venous thromboembolism. For cases, prescriptions for warfarin in the six weeks before diagnosis of venous thromboembolism did not trigger exclusion as they could plausibly be treatment for the index event itself. Cases in which there was any use of warfarin earlier than this six week period, however, were excluded.

\section{Data}

We extracted demographic information including year of birth, sex, and fifths of Townsend score (a measure of socioeconomic status), together with the most recent body mass index (BMI) and smoking status before the index date. Individuals were coded as having schizophrenia, bipolar disorder, and/or dementia if these diagnoses were recorded before the index date. We extracted data recorded before the index date on comorbid conditions that could increase the risk of venous thromboembolism (coronary heart disease, cardiac failure, stroke, cancer, inflammatory bowel disease, liver disease, varicose veins, gastrointestinal bleed, Parkinson's disease, renal disease, asthma, diabetes, hypertension, hyperlipidaemia). ${ }^{1516}$ For several events associated with increased risk in the short term (hip surgery, hip or lower limb fractures, acute infections, pregnancy), ${ }^{11516}$ we extracted data for the six months before the index date. We also extracted information on whether there was any computer recorded evidence of a hospital admission in the preceding 31-183 days (admissions in the preceding 30 days might have been for the thrombosis itself) and included this in the analysis as a binary variable.

We assessed exposure to drugs on the basis of prescriptions on or before the index date. For the exposures of primary interest (antipsychotics, $B N F$, chapters 4.2 .1 and 4.2.2), full prescription data were collected, including drug name, formulation, dose instructions, and date. For some drugs that increase risk of venous thromboembolism (oral contraceptives and hormone replacement therapy, ${ }^{16} 17$ tamoxifen ${ }^{18}$ ) and other commonly used drugs (statins, non-steroidal anti-inflammatory drugs (NSAIDs), aspirin, antimanics), we recorded only whether any prescriptions were issued in the 24 months before the index date.

\section{Statistical methods}

The analyses were conducted in Stata version 9.2. Each individual was classified for exposure to antipsychotics as a current user (one or more prescriptions within three months before index date); recent user (four and 12 months before); past user (13 and 24 months before); or not exposed within the previous 24 months. We subclassified current users as new users (prescription for antipsychotic during the three months before the index date, after at least 12 months with no prescription for that antipsychotic) or as continuing users. Exposure during the past 24 months was also classified according to type (conventional only, atypical only, both) and potency (high potency only, low potency only, or both, with high potency defined as equivalent dose of more than $100 \mathrm{mg}$ chlorpromazine). When possible, we calculated the daily dose equivalent of each script and classified individuals as low dose only $(\leq 25 \%$ of maximum recommended in $B N F$ ), medium/low dose (under maximum), or any high dose (maximum and over). The number of different antipsychotic drugs prescribed to each individual, and the number of scripts each received, were ascertained.

Patients with more than one mental health indication were categorised according to a hierarchy: schizophrenia, bipolar disorder without schizophrenia, and dementia without schizophrenia or bipolar disorder. BMI values outside the range 15-50 were treated as missing.

\section{Case-control analyses}

We undertook multiple conditional logistic regression to estimate the odds ratio (with 95\% confidence interval) for risk of venous thromboembolism associated 
Table 1|Age-sex incidence rates for venous thromboembolism in QResearch primary care database

\begin{tabular}{|c|c|c|c|}
\hline Age (years) & $\begin{array}{l}\text { No of } \\
\text { events }\end{array}$ & $\begin{array}{l}\text { Person years } \\
\text { at risk }\end{array}$ & $\begin{array}{c}\text { Incidence/ } \\
100000 \text { person years }\end{array}$ \\
\hline \multicolumn{4}{|l|}{ Women: } \\
\hline $16-24$ & 248 & 767725 & 32 \\
\hline $25-34$ & 1381 & 2663258 & 52 \\
\hline $35-44$ & 1642 & 2733104 & 60 \\
\hline $45-54$ & 1887 & 2297636 & 82 \\
\hline $55-64$ & 2651 & 1948580 & 136 \\
\hline $65-74$ & 3523 & 1522939 & 231 \\
\hline $75-84$ & 4155 & 1176311 & 353 \\
\hline$\geq 85$ & 2107 & 522421 & 403 \\
\hline Total & 17594 & 13631973 & 129 \\
\hline \multicolumn{4}{|l|}{ Men: } \\
\hline $16-24$ & 94 & 737655 & 13 \\
\hline $25-34$ & 841 & 2651541 & 32 \\
\hline $35-44$ & 1354 & 2879488 & 47 \\
\hline $45-54$ & 1812 & 2392848 & 76 \\
\hline $55-64$ & 2784 & 1978988 & 141 \\
\hline $65-74$ & 3477 & 1394184 & 249 \\
\hline $75-84$ & 2812 & 813588 & 346 \\
\hline$\geq 85$ & 844 & 222328 & 380 \\
\hline Total & 14018 & 13070621 & 107 \\
\hline Total & 31612 & 26702594 & 118 \\
\hline
\end{tabular}

with any use of antipsychotics in the 24 months before the index date. The odds ratios are unbiased estimates of rate ratios as we used incidence density sampling to select controls. Odds ratios and 95\% confidence intervals were estimated for subgroups based on timing, type, potency, duration, and dose of antipsychotics and for individual antipsychotics with a sufficiently high level of use. We compared the risk for current antipsychotic users (prescriptions in the previous three months) between new users and continuing users.

The analyses were adjusted for socioeconomic status, the comorbidity and drug variables listed above, and the number of complete months of data before the index date. Adjustment was not initially made for smoking or BMI, because of missing data, but after we imputed missing values we repeated the analyses with these variables added to the models. We tested for interactions between use of antipsychotics and age, sex, socioeconomic status, BMI, smoking status, and category of mental health.

We repeated the main analyses twice. The first analysis was restricted to idiopathic venous thromboembolism, and cases and controls with major risk factors for venous thromboembolism (previous cancer, coronary heart disease, stroke, congestive cardiac failure; hip surgery, hip or lower limb fracture, or pregnancy within the previous six months) were excluded. We then excluded individuals with diagnoses of manic depression or schizophrenia.

We calculated the numbers needed to harm per year by applying the incidence of first venous thromboembolism and using the adjusted odds ratio from current use of antipsychotic drugs by type, potency, timing, quantity, and dose. We calculated this separately for all patients aged 16 and over and for those aged 65 and over. We also estimated the number of additional cases of venous thromboembolism expected per 10000 treated patients per year. We calculated approximate 95\% confidence intervals for these estimates.

\section{Multiple imputation}

Multiple imputation was carried out with the Stata ICE programs, ${ }^{19}$ with all the main analysis variables entered in the prediction equation, together with the matching variables of age, sex, and index year. Five imputed datasets were created and combined for analysis.

\section{RESULTS}

\section{Study population}

A total of 453 QResearch practices met the inclusion criteria. The study population consisted of 7267673 patients with 26702594 person years of observation. We identified 31612 incident cases of venous thromboembolism, an overall crude rate of 118 per 100000 person years (table 1, figure). Incidence rates among women rose from 32 per 100000 person years at age $16-24$ to 403 at age 85 and over; for men the corresponding numbers were 13 and 380 .

\section{Cases and controls}

The 31612 cases were matched to 125559 controls. We excluded 6016 cases (19\%) and 17595 controls (14\%), principally because they had less than 24 months of data before the index date (table 2). Cases without any matched controls, and controls without a matched case, were then excluded, leaving 25532 eligible cases (15975 with deep vein thrombosis and 9557 with pulmonary embolism) and 89491 eligible controls.

Table 3 shows that cases were more likely than controls to have a high BMI and marginally more likely to live in an area of socioeconomic deprivation. Overall, the prevalence of relevant mental health conditions

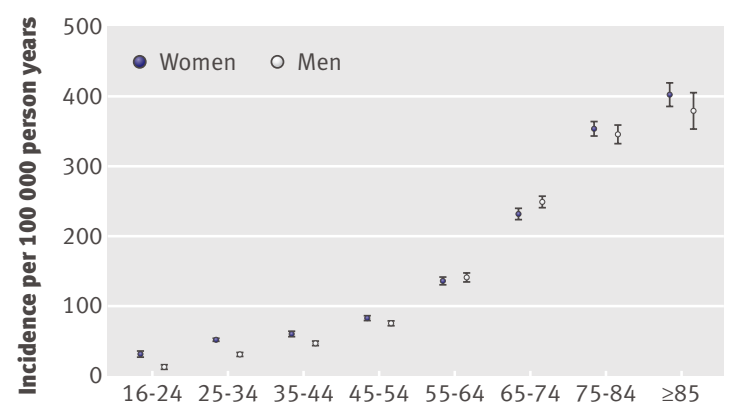

Age (years)

Incidence of venous thromboembolism per 100000 person years by sex and age, with error bars representing $95 \%$ confidence intervals 


$\begin{aligned} & \text { Table } 2 \text { | Eligibility of cases (patients with first ever record of venous thromboembolism) and } \\
& \text { matched controls. Figures are numbers (percentages) of individuals }\end{aligned}$
\begin{tabular}{lcc} 
& Cases & Controls \\
Extracted from QResearch & 31612 & 125559 \\
\hline Excluded: & $3648(11.5)$ & $11543(9.2)$ \\
\hline$\langle 24$ months of data before index date & $494(1.6)$ & $2590(2.1)$ \\
\hline Missing socioeconomic status & $1874(5.9)$ & $3462(2.8)$ \\
\hline Previous use of warfarin & $64(0.2)$ & $18473(14.7)$ \\
\hline Matched case or controls excluded & $25532(80.8)$ & $89491(71.3)$ \\
\hline Eligible
\end{tabular}

was $0.4 \%$ for schizophrenia, $0.3 \%$ for bipolar disorder, and $1.0 \%$ for dementia; eight cases and 31 controls had more than one of these. All the suggested risk factors for venous thromboembolism were more prevalent among cases than controls. For many conditions the difference was small, but there was a greater disparity for cancer and for recent fractures, hip surgery, and acute infections. The use of drugs that could increase risk of venous thromboembolism (oral contraceptives, hormone replacement therapy, tamoxifen) was more common among cases than controls.

\section{Risk associated with antipsychotics}

New users-Table 4 shows that 2126 cases (8.3\%) and 4752 controls $(5.3 \%)$ had received an antipsychotic drug in the previous 24 months. Overall, antipsychotic users had a $32 \%$ greater risk of venous thromboembolism than non-users (adjusted odds ratio 1.32, 95\% confidence interval 1.23 to 1.42). Among antipsychotic users, $38 \%$ were classed as current users (a script within the previous three months): their increase in risk was $56 \%(1.56,1.39$ to 1.75$)$ compared with $36 \%$ for recent users $(1.36,1.20$ to 1.54$)$; for past users the risk was not significantly increased (1.04, 0.91 to 1.18$)$. Among current antipsychotic users, 15\% had started a new drug within the three months before the index date, and this group of new users showed a greater increase in risk than continuing users $(1.97,1.66$ to 2.33 , for new users; $1.29,1.11$ to 1.51 , for continuing users).

Dose and type of antipsychotic - Table 4 also shows that $87 \%$ of controls $(4156 / 4752)$ of those prescribed antipsychotics had received only conventional drugs, $90 \%$ (4293/4752) had received only high potency drugs, and $51 \%(2404 / 4752)$ had received only a single prescription in the past 24 months; $6 \%$ (256) of those taking antipsychotics had changed to a different antipsychotic drug, and 1\% (44) had received the maximum daily dose (or greater) of any antipsychotic.

Atypicalv conventional-The risk was greater for individuals prescribed atypical drugs rather than conventional drugs (adjusted odds ratios 1.73, 1.37 to 2.17, for atypical; $1.28,1.18$ to 1.38 , for conventional). It was also greater for patients prescribed low rather than high potency drugs $(1.99,1.52$ to 2.62 , for low potency; $1.28,1.18$ to 1.38 , for high potency).

Number of prescriptions - The risk was marginally higher for individuals receiving more than 12 scripts in the past 24 months (adjusted odds ratio 1.44, 1.18 to 1.75 ), but those receiving only one had a significantly higher risk than those receiving none (1.32, 1.17 to 1.49 ).

Multiple drugs - Individuals prescribed two or more different antipsychotics had a greater risk than those receiving one (adjusted odds ratio 1.99, 1.49 to 2.65, for two or more drugs; $1.29,1.20$ to 1.39 , for one drug).

Dose response and route of delivery-There was an apparent dose-response gradient, but individuals receiving medium or high dose scripts were also more likely to have received two or more different drugs and to have received atypical drugs rather than conventional drugs. When we added the number of drugs or the types of antipsychotics to the models there was no significant relation between dose and risk. Individuals receiving any scripts for injections (short acting or depot) showed a substantially higher risk (adjusted odds ratio 3.24, 2.04 to 5.17) than individuals receiving only oral drugs or suppositories (1.30, 1.20 to 1.40$)$, but the numbers receiving injections were small.

Individual drugs - The most commonly prescribed drug was prochlorperazine, a high potency phenothiazine, which, in addition to treating psychosis, is commonly prescribed for nausea, vomiting, and vertigo. Prochlorperazine was prescribed for 5133 individuals, $75 \%$ of the total number who had taken antipsychotics, and for 3243 of these only a single script had been given (not tabulated). The other most commonly prescribed drugs were (in order) risperidone, haloperidol, olanzapine, chlorpromazine, trifluoperazine, and quetiapine (table4). Separate odds ratios were estimated for exposure to these drugs, and the highest risks were found among individuals prescribed quetiapine, chlorpromazine, and haloperidol (adjusted odds ratio 2.81 (1.75 to 4.50$), 1.77$ (1.27 to 2.48 ), and 2.17 (1.55 to $3.02)$, respectively).

Sensitivity analyses - All the results were similar when we carried out analyses on individuals having excluded those with either schizophrenia or manic depression and having excluded those with major risk factors for venous thromboembolism (see appendix on bmj.com for full results).

Missing data -Data on smoking or BMI, or both, were missing for 6534 cases $(25.6 \%)$ and 25720 controls (28.7\%): they were marginally more likely to be men and to live in an area of social deprivation than those with complete data. Odds ratios based on the imputed datasets, with smoking and BMI added to the adjustment variables, were close to the ones reported in table 4. For example, the overall odds ratios after imputation were 1.32 (1.24 to 1.40$)$ for any antipsychotic use in the previous 24 months, 1.57 (1.43 to 1.72 ) for current use, 1.31 (1.19 to 1.46 ) for recent use, and 1.06 (0.95 to 1.18) for past use.

Confounding variables - Table 5 shows associations between risk of venous thromboembolism and the variables treated as potential confounders, adjusted for each other and for any use of antipsychotics in the previous 24 months. We used the imputed datasets so that smoking and BMI could be included, and the table 
Table 3 |Characteristics of cases (patients with first ever record of venous thromboembolism) and matched controls at index date. Figures are numbers (percentages) unless stated otherwise

\begin{tabular}{|c|c|c|}
\hline Characteristic & Cases $(n=25532)$ & Controls $(n=89491)$ \\
\hline Men & $11318(44.3)$ & 39521 (44.2) \\
\hline Women & $14214(55.7)$ & $49970(55.8)$ \\
\hline Median (IQR) age (years) & $67(53-77)$ & $67(53-77)$ \\
\hline \multicolumn{3}{|l|}{ Smoking status: } \\
\hline Current smoker & $4701(18.4)$ & $14901(16.7)$ \\
\hline Not current smoker & $17531(68.7)$ & $59130(66.1)$ \\
\hline Smoking status not recorded & $3300(12.9)$ & $15460(17.3)$ \\
\hline \multicolumn{3}{|l|}{ Body mass index (BMI): } \\
\hline Not overweight (15.0-24.9) & $6454(25.3)$ & 28108 (31.4) \\
\hline Overweight (25.0-29.9) & $7390(28.9)$ & $24869(27.8)$ \\
\hline Obese (30-50) & $5629(22.0)$ & $12527(14.0)$ \\
\hline No valid BMI recorded & $6059(23.7)$ & $23987(26.8)$ \\
\hline \multicolumn{3}{|l|}{ Fifth of socioeconomic status: } \\
\hline 1 (least deprived) & $5798(22.7)$ & $21898(24.5)$ \\
\hline 2 & $5110(20.0)$ & $19205(21.5)$ \\
\hline 3 & $5233(20.5)$ & $18592(20.8)$ \\
\hline 4 & $5178(20.3)$ & $16868(18.8)$ \\
\hline 5 (most deprived) & $4213(16.5)$ & $12928(14.4)$ \\
\hline Median (IQR) months of previous data & $164(91-297)$ & $167(94-303)$ \\
\hline \multicolumn{3}{|l|}{ Mental health conditions*: } \\
\hline Schizophrenia & $121(0.5)$ & $325(0.4)$ \\
\hline Bipolar disorder & $73(0.3)$ & $198(0.2)$ \\
\hline Dementia & $327(1.3)$ & $826(0.9)$ \\
\hline None of the above & $25011(98.0)$ & $88142(98.5)$ \\
\hline \multicolumn{3}{|l|}{ Comorbidities: } \\
\hline Coronary heart disease & $3502(13.7)$ & $8982(10.0)$ \\
\hline Congestive cardiac failure & $1553(6.1)$ & $2546(2.8)$ \\
\hline Stroke or transient ischaemic attack & $1861(7.3)$ & $4235(4.7)$ \\
\hline Any cancer & $3613(14.2)$ & $4119(4.6)$ \\
\hline Inflammatory bowel disease & $349(1.4)$ & $715(0.8)$ \\
\hline Chronic liver disease & $134(0.5)$ & $279(0.3)$ \\
\hline Varicose veins & $1096(4.3)$ & $2380(2.7)$ \\
\hline Peptic ulcer with complications & $586(2.3)$ & $1330(1.5)$ \\
\hline Parkinson's disease & $241(0.9)$ & $563(0.6)$ \\
\hline Chronic renal disease & $300(1.2)$ & $421(0.5)$ \\
\hline Asthma & $3225(12.6)$ & $7869(8.8)$ \\
\hline Diabetes & $1951(7.6)$ & $5774(6.5)$ \\
\hline Hypertension & $7671(30.0)$ & $24291(27.1)$ \\
\hline Hyperlipidaemia & $1458(5.7)$ & $4696(5.2)$ \\
\hline \multicolumn{3}{|l|}{ Events in previous six months: } \\
\hline Hip operation & $802(3.1)$ & $228(0.3)$ \\
\hline Leg or hip fracture & $806(3.2)$ & $224(0.3)$ \\
\hline Acute infection & $4201(16.5)$ & $7022(7.8)$ \\
\hline Pregnancy & $390(1.5)$ & $427(0.5)$ \\
\hline Hospital admission in past 31-183 days & $965(3.7)$ & $1238(1.38)$ \\
\hline \multicolumn{3}{|l|}{ Use of medications in previous 24 months: } \\
\hline Statins & $3279(12.8)$ & $10760(12.0)$ \\
\hline NSAIDs (traditional or COX 2) & $13574(53.2)$ & $34343(38.4)$ \\
\hline Aspirin & $5659(22.2)$ & $15829(17.7)$ \\
\hline Oral contraceptive (combined or progestogen only) & $1088(4.3)$ & $2930(3.3)$ \\
\hline Hormone replacement therapy & $1402(5.5)$ & $4015(4.5)$ \\
\hline Tamoxifen & $651(2.5)$ & $738(0.8)$ \\
\hline Antimanics & $322(1.3)$ & $574(0.6)$ \\
\hline
\end{tabular}

$\mathrm{IQR}=$ interquartile range; $\mathrm{BMI}=$ body mass index; NSAIDs=non-steroidal anti-inflammatory drugs; COX 2=cyclooxygenase-2 selective.

*Patients with more than one mental health diagnosis shown in one group only according to hierarchy of schizophrenia, bipolar disorder, dementia. shows significant associations with risk for both these variables and socioeconomic status. Individuals with a diagnosis of dementia were at higher risk than those with schizophrenia, bipolar disorder, or none of these conditions. Most of the comorbidities and health events considered as potential risk factors were associated with a relatively small increase in risk, but there was a more than threefold increase associated with cancer and about a 13-fold increase associated with recent surgery or fractures. Individuals prescribed statins or aspirin in the past 24 months had a lower risk of venous thromboembolism, and those prescribed NSAIDS, oral contraceptives, hormone replacement therapy, tamoxifen, or anti-manics had a higher risk.

Interactions - Inclusion of interaction terms showed that the risk associated with antipsychotic use did not differ significantly between subgroups based on age, sex, socioeconomic status, BMI, or mental health category. Smokers had a higher risk related to antipsychotics than non-smokers: the adjusted odds ratio for antipsychotic use was 1.68 (1.45 to 1.93) among smokers and 1.27 (1.18 to 1.36) among non-smokers.

Numbers needed to harm-Table 6 shows the numbers needed to treat to harm $(\mathrm{NNH})$ for each category of antipsychotic use and the number of excess cases per 10000 patients treated over a year for all patients aged over 16 and for those aged 65 years and older. For example, the NNH for any antipsychotic use in the past 24 months for patients aged 65 year and older was 1044 (795 to 1452); for new users in the past three months it was 344 (251 to 506); and for continuing users it was 1152 (655 to 3037). The corresponding numbers of excess cases of venous thromboembolism per 10000 treated patients were 10 (7 to 13), 29 (20 to 40), and 9 (3 to 15$)$, respectively.

\section{DISCUSSION}

\section{Summary of main findings}

This nested case-control study, based on the primary care clinical records of 115000 people in the UK, found a $32 \%$ increased risk of venous thromboembolism for individuals prescribed antipsychotic drugs in the past 24 months. The increase in risk was $56 \%$ for individuals with any antipsychotic use in the past three months, and $97 \%$ for those who had newly started on an antipsychotic in the past three months. The absolute risks, however, were low, with an excess of four extra cases of venous thromboembolism per 10000 patients treated over one year in patients of all ages, and 10 for patients aged 65 and over.

\section{Methodological considerations and strengths}

The study was based on the primary care records of a large and representative population cohort, avoiding bias from selection, non-response, or poor recall. General practices in the UK have good levels of accuracy and completeness in recording clinical diagnoses and prescribed drugs,${ }^{20}$ and practices that contribute to primary care databases such as QResearch are thought to perform even better. ${ }^{21}$ The recorded clinical diagnoses 
Table 4 |Complete case analysis for exposure to antipsychotic medication in past 24 months, and odds ratios for venous thromboembolism associated with antipsychotic use in all patients

\begin{tabular}{|c|c|c|c|c|}
\hline Antipsychotic exposure & $\begin{array}{c}\text { No }(\%) \text { of cases } \\
\quad(n=25532)\end{array}$ & $\begin{array}{c}\text { No }(\%) \text { of controls } \\
(n=89491)\end{array}$ & $\begin{array}{c}\text { Unadjusted odds ratio } \\
\text { and } 95 \% \mathrm{Cl}\end{array}$ & $\begin{array}{c}\text { Adjusted* odds ratio } \\
(95 \% \mathrm{Cl})\end{array}$ \\
\hline \multicolumn{5}{|l|}{ Any antipsychotic use: } \\
\hline No§ & 23406 (91.7) & $84739(94.7)$ & 1.00 & 1.00 \\
\hline Yes & $2126(8.3)$ & $4752(5.3)$ & $1.62(1.52$ to 1.73$)$ & $1.32(1.23$ to 1.42$)$ \\
\hline \multicolumn{5}{|l|}{ Timing of antipsychotic use: } \\
\hline Current (within past 3 months) & $910(3.6)$ & 1719 (1.9) & 1.95 (1.76 to 2.16$)$ & $1.56(1.39$ to 1.75$)$ \\
\hline New user & $440(1.7)$ & $585(0.7)$ & 2.55 (2.19 to 2.97$)$ & 1.97 (1.66 to 2.33$)$ \\
\hline Continuing user & $470(1.8)$ & $1134(1.3)$ & 1.59 (1.39 to 1.82$)$ & $1.29(1.11$ to 1.51$)$ \\
\hline Recent (4-12 months before) & $677(2.7)$ & $1481(1.7)$ & $1.68(1.50$ to 1.88$)$ & $1.36(1.20$ to 1.54$)$ \\
\hline Past (13-24 months before) & $539(2.1)$ & $1552(1.7)$ & 1.23 (1.09 to 1.38$)$ & 1.04 (.911 to 1.18$)$ \\
\hline \multicolumn{5}{|l|}{ Types of antipsychotic received: } \\
\hline Conventional only & $1817(7.1)$ & $4156(4.6)$ & $1.58(1.48$ to 1.70$)$ & 1.28 (1.18 to 1.38$)$ \\
\hline Atypical only & $221(0.9)$ & $462(0.5)$ & $1.74(1.42$ to 2.14$)$ & $1.73(1.37$ to 2.17$)$ \\
\hline Conventional and atypical & $88(0.3)$ & $134(0.1)$ & 2.47 (1.71 to 3.57$)$ & 1.77 (1.16 to 2.69$)$ \\
\hline \multicolumn{5}{|l|}{ Potency of antipsychotics received: } \\
\hline Low potency only & $162(0.6)$ & $316(0.4)$ & 2.51 (1.96 to 3.20$)$ & 1.99 (1.52 to 2.62$)$ \\
\hline High potency only & $1875(7.3)$ & $4293(4.8)$ & $1.55(1.45$ to 1.66$)$ & 1.28 (1.18 to 1.38$)$ \\
\hline Low and high potency & $89(0.3)$ & $143(0.2)$ & $2.31(1.65$ to 3.24$)$ & 1.67 (1.14 to 2.45$)$ \\
\hline \multicolumn{5}{|l|}{ No of antipsychotic scripts received: } \\
\hline 1 & $1092(4.3)$ & $2404(2.7)$ & 1.59 (1.46 to 1.74$)$ & $1.30(1.18$ to 1.43$)$ \\
\hline $2-12$ & $729(2.9)$ & $1639(1.8)$ & $1.63(1.46$ to 1.82$)$ & $1.32(1.17$ to 1.49$)$ \\
\hline$\geq 13$ & $305(1.2)$ & $709(0.8)$ & $1.70(1.43$ to 2.01$)$ & 1.44 (1.18 to 1.75$)$ \\
\hline \multicolumn{5}{|l|}{ No of different antipsychotic drugs received: } \\
\hline 1 & $1952(7.6)$ & $4496(5.0)$ & 1.57 (1.46 to 1.68$)$ & 1.29 (1.20 to 1.39$)$ \\
\hline$\geq 2$ & $174(0.7)$ & $256(0.3)$ & $2.73(2.11$ to 3.52$)$ & 1.99 (1.49 to 2.65$)$ \\
\hline \multicolumn{5}{|l|}{ Dose of received: } \\
\hline Low dose scripts only ( $\leq 25 \%$ maximum) & $1832(7.2)$ & $4237(4.7)$ & 1.56 (1.46 to 1.67$)$ & $1.26(1.17$ to 1.36$)$ \\
\hline Medium dose scripts† & $152(0.6)$ & $270(0.3)$ & 1.93 (1.51 to 2.48$)$ & 1.68 (1.27 to 2.23$)$ \\
\hline High dose scripts ( $\geq$ maximum) $\ddagger$ & $27(0.1)$ & $44(0.0)$ & 2.63 (1.41 to 4.90$)$ & 2.59 (1.27 to 5.29$)$ \\
\hline No scripts with calculable daily dose & $115(0.5)$ & $201(0.2)$ & $2.35(1.75$ to 3.15$)$ & 2.15 (1.56 to 2.98$)$ \\
\hline \multicolumn{5}{|l|}{ Mode of delivery: } \\
\hline Non-injection modes only & $2057(8.1)$ & $4690(5.2)$ & $1.58(1.48$ to 1.69$)$ & 1.30 (1.20 to 1.40$)$ \\
\hline Any by injection & $69(0.3)$ & $62(0.1)$ & 4.56 (2.98 to 6.98$)$ & $3.24(2.04$ to 5.17$)$ \\
\hline Prochlorperazine (conventional, high potency) & $1525(6.0)$ & $3608(4.0)$ & $1.50(1.40$ to 1.62$)$ & $1.22(1.13$ to 1.33$)$ \\
\hline Risperidone (atypical, high potency) & $148(0.6)$ & $293(0.3)$ & 1.41 (1.07 to 1.85$)$ & $1.24(.916$ to 1.68$)$ \\
\hline Haloperidol (conventional, high potency) & $149(0.6)$ & $203(0.2)$ & $3.36(2.50$ to 4.50$)$ & 2.17 (1.55 to 3.02$)$ \\
\hline Olanzapine (atypical, high potency) & $102(0.4)$ & $223(0.2)$ & $1.63(1.21$ to 2.20$)$ & 1.49 (1.07 to 2.08$)$ \\
\hline Chlorpromazine (conventional, low potency) & $113(0.4)$ & $184(0.2)$ & 2.25 (1.67 to 3.02$)$ & 1.77 (1.27 to 2.48$)$ \\
\hline Trifluoperazine (conventional, high potency) & $82(0.3)$ & $156(0.2)$ & 1.61 (1.13 to 2.29$)$ & $1.27(0.86$ to 1.87$)$ \\
\hline Quetiapine (atypical, low potency) & $58(0.2)$ & $90(0.1)$ & $3.64(2.38$ to 5.56$)$ & $2.81(1.75$ to 4.50$)$ \\
\hline
\end{tabular}

*Adjusted for socioeconomic status, mental health indication, months of data, coronary heart disease, congestive heart failure, stroke or transient ischaemic attack, cancer, inflammatory bowel disease, liver disease, varicose veins, peptic ulcer with complications, Parkinson's disease, chronic renal disease, asthma, diabetes, hypertension, hyperlipidaemia, hip operation, fracture, pregnancy, acute infection, hospital admission in past 31-183 days, statins, NSAIDs, aspirin, oral contraceptives, hormone replacement therapy, tamoxifen, antimanic drug. †Includes individuals with both medium and low dose scripts.

flndividuals in high dose category might also have received medium or low dose scripts, or both.

$\S$ Reference category for all odds ratios.

of venous thromboembolism were not independently verified for the study, but in other studies the inclusion of "possible" venous thromboembolism cases did not alter the estimates based on confirmed cases. ${ }^{1422}$ The observed incidence rate in our population was close to the 117 per 100000 reported elsewhere. $^{23}$

We adjusted for many potential confounding factors, including comorbidities, health events, and drugs. We carried out an alternative analysis restricted to individuals with no major risk factors for venous throm boembolism because inclusion of non-idiopathic cases can bias an estimate towards the null. ${ }^{24} \mathrm{We}$ found little difference between the estimates based on all individuals (with adjustment for risk factors) and those based on individuals without major risk factors. Clinicians prescribing antipsychotics might have no current perception of thrombotic risk and therefore do not take an individual's other thrombotic risk factors into 
Table $5 \mid$ Odds ratios for venous thromboembolism associated with potential confounding variables based on multiply imputed data

\begin{tabular}{|c|c|c|}
\hline Exposure/confounder & Adjusted odds ratio* $(95 \% \mathrm{Cl})$ & $P$ value \\
\hline \multicolumn{3}{|l|}{ Socioeconomic status (fifth of Townsend score): } \\
\hline 1 (least deprived) & 1.0 & \\
\hline 2 & 0.98 (0.93 to 1.03$)$ & 0.365 \\
\hline 3 & $1.03(0.98$ to 1.08$)$ & 0.204 \\
\hline 4 & $1.10(1.05$ to 1.16$)$ & $<0.001$ \\
\hline 5 (most deprived) & 1.19 (1.12 to 1.27$)$ & $<0.001$ \\
\hline \multicolumn{3}{|l|}{ Smoking: } \\
\hline Not current smoker & 1.0 & \\
\hline Current smoker & 1.31 (1.24 to 1.39$)$ & $<0.001$ \\
\hline \multicolumn{3}{|l|}{ Body mass index (BMI): } \\
\hline $15-24.9$ & 1.0 & \\
\hline 25-29.9 (overweight) & 1.31 (1.25 to 1.36$)$ & $<0.001$ \\
\hline $30-50$ (obese) & $1.92(1.83$ to 2.01$)$ & $<0.001$ \\
\hline \multicolumn{3}{|l|}{ Mental health conditions: } \\
\hline None & 1.0 & \\
\hline Schizophrenia & 1.34 (1.15 to 1.55$)$ & $<0.001$ \\
\hline Bipolar disorder & $1.22(0.91$ to 1.65$)$ & 0.186 \\
\hline Dementia & $1.20(0.95$ to 1.51$)$ & 0.126 \\
\hline \multicolumn{3}{|l|}{ Comorbidities and health events: } \\
\hline Coronary heart disease & 1.34 (1.15 to 1.55$)$ & $<0.001$ \\
\hline Congestive cardiac failure & $1.22(0.91$ to 1.65$)$ & $<0.001$ \\
\hline Stroke or transient ischaemic attack & $1.20(0.95$ to 1.51$)$ & $<0.001$ \\
\hline Any cancer & 1.34 (1.15 to 1.55$)$ & $<0.001$ \\
\hline Inflammatory bowel disease & $1.22(0.91$ to 1.65$)$ & $<0.001$ \\
\hline Chronic liver disease & $1.20(0.95$ to 1.51$)$ & 0.002 \\
\hline Varicose veins & 1.34 (1.15 to 1.55$)$ & $<0.001$ \\
\hline Peptic ulcer with complications & $1.22(0.91$ to 1.65$)$ & $<0.001$ \\
\hline Parkinson's disease & $1.20(0.95$ to 1.51$)$ & $<0.001$ \\
\hline Chronic renal disease & 1.34 (1.15 to 1.55$)$ & $<0.001$ \\
\hline Asthma & $1.22(0.91$ to 1.65$)$ & $<0.001$ \\
\hline Diabetes & $1.20(0.95$ to 1.51$)$ & 0.274 \\
\hline Hypertension & 1.34 (1.15 to 1.55$)$ & 0.154 \\
\hline Hyperlipidaemia & $1.22(0.91$ to 1.65$)$ & 0.354 \\
\hline Hip operation in past 6 months & $1.20(0.95$ to 1.51$)$ & $<0.001$ \\
\hline Leg or hip fracture in past 6 months & 1.34 (1.15 to 1.55$)$ & $<0.001$ \\
\hline Pregnancy in past 6 months & $1.22(0.91$ to 1.65$)$ & $<0.001$ \\
\hline Acute infection in past 6 months & $1.20(0.95$ to 1.51$)$ & $<0.001$ \\
\hline Hospital admission in past $31-183$ days & 1.34 (1.15 to 1.55$)$ & $<0.001$ \\
\hline \multicolumn{3}{|l|}{ Use of other medications in past 24 months: } \\
\hline Statins & $0.78(0.73$ to 0.83$)$ & $<0.001$ \\
\hline NSAIDs (traditional or COX 2) & $1.78(1.71$ to 1.84$)$ & $<0.001$ \\
\hline Aspirin & $0.76(0.72$ to 0.80$)$ & $<0.001$ \\
\hline Oral contraceptive (combined or progestogen only) & $1.33(1.21$ to 1.47$)$ & $<0.001$ \\
\hline Hormone replacement therapy & $1.33(1.23$ to 1.44$)$ & $<0.001$ \\
\hline Tamoxifen & 1.48 (1.31 to 1.68$)$ & $<0.001$ \\
\hline Antimanics & 1.62 (1.39 to 1.89$)$ & $<0.001$ \\
\hline
\end{tabular}

NSAIDs=non-steroidal anti-inflammatory drugs; COX 2=cyclo-oxygenase-2 selective.

*Adjusted for all other variables in table, plus number of months of data and any use of antipsychotics in previous 24 months.
Another strength of our study was that we had access to full prescription details for all antipsychotics, including drug name and formulation, dose instructions, and dates. This enabled us to look in detail at characteristics of the drug exposure in relation to risk. It also allowed for a comparison of risk between new users and those who had taken antipsychotic drugs for longer. Inclusion of all prevalent users can underestimate risks, which occur soon after the start of treatment, ${ }^{25}$ and we found that the risk was highest in the early months, in common with some previous studies. ${ }^{59}$

\section{Study limitations}

As with all observational studies, our study has limitations. About a quarter of the records had missing data on smoking status or BMI, or both. By imputing values for these variables we were able to ascertain that including them in the models had almost no effect on the estimates. There might have been some confounding by indication as in most patients we were unable to ascertain the reason for prescription of antipsychotics. This makes it difficult to distinguish the effects of the drugs from any effect of the condition for which the drug was prescribed. When we excluded people with schizophrenia or manic depression from the analyses, however, the results were largely unchanged. There could still be residual confounding and the true effect of this group of drugs is probably less than has been estimated in this study.

Our study was based on patients who have access to primary care and excluded any individuals not registered with a general practitioner-for example, those in prisons. In the overall database the most deprived socioeconomic group is marginally under-represented. Even in a dataset of this size, the number of cases and controls exposed to individual drugs was often too small to estimate separate odds ratios. The possibility of residual confounding by indication cannot be ruled out and is a further barrier to making definitive risk comparisons between different drugs. Furthermore, our study wasn't designed to and is not able to offer hypotheses on individual mechanisms in the genesis of thromboembolism for each drug. In terms of the overall findings, however, the fact that past users of antipsychotics showed no significantly increased risk argues that the drugs, rather than any fixed characteristics of those prescribed them, are responsible for the increased risk in current users.

We have presented numbers needed to harm and number of estimated excess cases per 10000 patients, which provides useful information at a population level. With this study design we were unable to derive individualised measures of absolute risk, which would take account of the patient's characteristics.

\section{Study in context of previous studies}

Previous studies on antipsychotics and venous thromboembolism had substantial differences in sample and design and produced highly disparate findings. Our case-control study found smaller estimated effects excluding patients with a recorded diagnosis of schizophrenia or manic depression and again found little difference compared with the estimates based on all individuals. 
Table $6 \mid$ Numbers needed to harm and excess additional cases of each outcome per 10000 patients prescribed antipsychotics over one year

\begin{tabular}{|c|c|c|c|c|}
\hline \multirow[b]{2}{*}{ Antipsychotic exposure } & \multicolumn{2}{|c|}{$\begin{array}{l}\text { Numbers needed to harm } \\
\qquad(95 \% \mathrm{Cl})\end{array}$} & \multicolumn{2}{|c|}{$\begin{array}{c}\text { Extra cases per } 10000 \text { treated } \\
(95 \% \mathrm{Cl})\end{array}$} \\
\hline & All ages & Age $\geq 65$ & All ages & Age $\geq 65$ \\
\hline Any antipsychotic use in past 24 months & $2640(2011$ to 3673$)$ & 1044 (795 to 1452$)$ & $4(3$ to 5$)$ & 10 (7 to 13$)$ \\
\hline \multicolumn{5}{|l|}{ Timing of antipsychotic use: } \\
\hline Current (within last 3 months) & 1508 (1126 to 2166$)$ & 597 (445 to 857) & 7 (5 to 9) & 17 (12 to 22$)$ \\
\hline New user of antipsychotics within past 3 months & $871(635$ to 1280$)$ & 344 (251 to 506$)$ & 11 (8 to 16$)$ & 29 (20 to 40$)$ \\
\hline $\begin{array}{l}\text { Continuing user of antipsychotics within past } \\
3 \text { months }\end{array}$ & 2913 (1656 to 7679$)$ & 1152 (655 to 3037$)$ & $3(1$ to 6$)$ & 9 (3 to 15$)$ \\
\hline Recent (4-12 months before) & 2346 (1564 to 4223$)$ & 928 (619 to 1670$)$ & $4(2$ to 6$)$ & 11 (6 to 16$)$ \\
\hline Past (13-24 months before) & NS & NS & NS & NS \\
\hline \multicolumn{5}{|l|}{ Types of antipsychotic received in past 24 months: } \\
\hline Conventional only & 3017 (2223 to 4693) & 1193 (879 to 1856$)$ & $3(2$ to 4$)$ & $8(5$ to 11$)$ \\
\hline Atypical only & 1157 (722 to 2283$)$ & 458 (286 to 903$)$ & $9(4$ to 14$)$ & 22 (11 to 35) \\
\hline Conventional and atypical & 1097 (500 to 5279 ) & 434 (198 to 2088) & $9(2$ to 20$)$ & 23 (5 to 51$)$ \\
\hline \multicolumn{5}{|l|}{ Potency of antipsychotics received in past 24 months: } \\
\hline Low potency only & $853(521$ to 1624$)$ & 337 (206 to 642) & 12 (6 to 19$)$ & 30 (16 to 48$)$ \\
\hline High potency only & 3017 (2223 to 4693) & 1193 (879 to 1856$)$ & $3(2$ to 4$)$ & $8(5$ to 11$)$ \\
\hline Low and high potency & 1261 (583 to 6034) & 499 (230 to 2386) & $8(2$ to 17$)$ & 20 (4 to 43$)$ \\
\hline \multicolumn{5}{|l|}{ No of antipsychotic scripts received in past 24 months: } \\
\hline 1 & 2816 (1964 to 4693) & 1114 (777 to 1856$)$ & $4(2$ to 5$)$ & 9 (5 to 13$)$ \\
\hline $2-12$ & 2640 (1724 to 4969$)$ & 1044 (682 to 1965$)$ & $4(2$ to 6$)$ & 10 (5 to 15$)$ \\
\hline$\geq 13$ & 1920 (1126 to 4693) & 759 (445 to 1856$)$ & 5 (2 to 9$)$ & 13 (5 to 22$)$ \\
\hline \multicolumn{5}{|c|}{ No of different antipsychotic drugs received in past 24 months: } \\
\hline 1 & 2913 (2166 to 4223) & $1152(857$ to 1670$)$ & $3(2$ to 5$)$ & $9(6$ to 12$)$ \\
\hline$\geq 2$ & $853(512$ to 1724$)$ & 337 (202 to 682) & $12(6$ to 20$)$ & 30 (15 to 49) \\
\hline \multicolumn{5}{|l|}{ Dose of antipsychotics received in past 24 months: } \\
\hline Low dose scripts only ( $\leq 25 \%$ maximum) & 3249 (2346 to 4969$)$ & 1285 (928 to 1965$)$ & $3(2$ to 4$)$ & $8(5$ to 11$)$ \\
\hline Medium dose scripts & 1242 (687 to 3129$)$ & $491(272$ to 1237$)$ & $8(3$ to 15$)$ & 20 (8 to 37$)$ \\
\hline High dose scripts ( $\geq$ maximum) & 531 (197 to 3129) & 210 (78 to 1237$)$ & 19 (3 to 51$)$ & 48 (8 to 128$)$ \\
\hline No scripts with calculable daily dose & 735 (427 to 1508) & 290 (169 to 597) & 14 (7 to 23$)$ & 34 (17 to 59$)$ \\
\hline \multicolumn{5}{|c|}{ Mode of delivery of antipsychotics received in past 24 months: } \\
\hline Non-injection modes only & 2816 (2112 to 4223$)$ & 1114 (835 to 1670$)$ & $4(2$ to 5$)$ & $9(6$ to 12$)$ \\
\hline Any by injection & 377 (203 to 812$)$ & $149(80$ to 321$)$ & 27 (12 to 49$)$ & $67(31$ to 125$)$ \\
\hline \multicolumn{5}{|l|}{ Individual antipsychotics received in past 24 months: } \\
\hline Prochlorperazine (conventional, high potency) & 3840 (2560 to 6498) & 1518 (1012 to 2570$)$ & $3(2$ to 4$)$ & $7(4$ to 10$)$ \\
\hline Risperidone (atypical, high potency) & NS & NS & NS & NS \\
\hline Haloperidol (conventional, high potency) & $722(418$ to 1536$)$ & 286 (165 to 607$)$ & 14 (7 to 24$)$ & 35 (16 to 60$)$ \\
\hline Olanzapine (atypical, high potency) & 1724 (782 to 12067$)$ & 682 (309 to 4772) & $6(1$ to 13$)$ & 15 (2 to 32$)$ \\
\hline Chlorpromazine (conventional, low potency) & 1097 (571 to 3129) & 434 (226 to 1237$)$ & 9 (3 to 18$)$ & 23 (8 to 44$)$ \\
\hline Trifluoperazine (conventional, high potency) & NS & NS & NS & NS \\
\hline Quetiapine (atypical, low potency) & 467 (241 to 1126$)$ & 185 (95 to 445$)$ & 21 (9 to 41$)$ & $54(22$ to 105$)$ \\
\hline
\end{tabular}

NS=non-significant.

than previous case-control studies, ${ }^{5-7}$ but our sample was much larger and more representative of the general population. Previous cohort studies were based on more restricted samples (such as older people, ${ }^{8}$ residents of nursing homes, ${ }^{9}$ and current or past users of clozapine $\left.^{10}\right)$. The two studies based on older people produced conflicting findings, one finding a significant association only for a subgroup using conventional drugs,${ }^{8}$ and the other an association for atypical drugs but not conventional drugs (though the latter were being prescribed at lower doses than previously found to increase risk). ${ }^{9}$ In our study, the risk was higher for individuals taking atypical drugs than for those taking conventional drugs. Our findings of a greater risk for individuals prescribed low rather than high potency drugs, and of no dose-response relation that could not be explained by other characteristics of the drug exposure, agree with the limited previous evidence. $^{56}$

\section{What this study adds}

Our study adds to the accumulating evidence of adverse health events associated with antipsychotic 


\section{WHAT IS ALREADY KNOWN ON THIS TOPIC}

Antipsychotic drugs, some of which are also prescribed for nausea, vomiting, and vertigo, have been associated with an increased risk of venous thromboembolism

Previous studies have been small, restricted to certain population groups, or have not covered newer atypical antipsychotic drugs

\section{WHAT THIS STUDY ADDS}

In a large primary care population there was an association between use of antipsychotic drugs and risk of venous thromboembolism

The increased risk was more marked among new users and those prescribed atypical antipsychotic drugs

Though these findings add to the accumulating evidence of adverse health events associated with antipsychotic drugs, they should be confirmed with other data sources

drugs. Recent studies have confirmed the significantly increased risks of serious events and mortality among people with dementia who are treated for behavioural problems with these drugs. ${ }^{2627}$ Nearly all use of antipsychotics in the primary care population we studied was for conditions such as nausea, vomiting, and vertigo, implying that the message on potential risk could have important implications for general practice. An increased risk of stroke associated with antipsychotics has recently been confirmed in primary care patients. ${ }^{28}$ Our findings would need to be replicated on another database before changes in clinical practice can be recommended, and larger numbers would be required to estimate the risks associated with individual antipsychotics. A study such as a self controlled case series analysis, which controls for all known and unknown confounders provided they are constant over time,,$^{29}$ would strengthen the evidence we found. ${ }^{29}$

If other studies replicate these findings, antipsychotic drugs should be used more cautiously for nausea and agitation, etc, especially among patients at high risk of thromboembolism. Patients need information on the balance of risks and benefits of these drugs before they start treatment. To achieve this, we need new algorithms designed to estimate a patient's absolute risk of thromboembolism that take account of individual level factors such as age, sex, deprivation, smoking, comorbidities, and concurrent drugs. These can then be used to explain the likely risk-benefit ratio to patients considering antipsychotic treatment as well as to identify those at highest risk who are likely to need closer monitoring or interventions to reduce their level of risk.

We acknowledge the contribution of EMIS and EMIS practices contributing to the QResearch database.

Contributors: $\mathrm{CP}$ contributed to the design, reviewed the literature, undertook the primary analysis, contributed to the interpretation, and wrote the first and subsequent drafts of the paper. CC contributed to the development of the protocol, design and analysis, and interpretation and drafting of the paper. JH-C initiated the study, contributed to the design, obtained approvals, prepared the data, and checked and added to the analysis, interpretation, and drafting of the paper. All authors approved the final draft of the paper originally submitted to the $B M J . J H-C$ and $C C$ approved the final draft of the paper revised after referees' comments as $\mathrm{CP}$ had retired. JH-C is guarantor.
Funding: This research received no specific grant from any funding agency in the public, commercial, or not-for-profit sectors.

Competing interests: All authors have completed the Unified Competing Interest form at www.icmje.org/coi_disclosure.pdf (available on request from the corresponding author) and declare no support from any organisation for the submitted work. JHC is codirector of QResearch, a not-for-profit organisation that is a joint partnership between the University of Nottingham and EMIS. EMIS is the leading supplier of IT for $60 \%$ of UK general practices. This work and any views expressed within it are solely those of the co-authors and not of any affiliated bodies or organisations.

Ethical approval: The proposal was approved by the Trent multicentre research ethics committee.

Data sharing: The patient level data from the QResearch are specifically licensed according to its governance framework. See www.gresearch.org for further details. The Read codes groups used are available from the authors on request.

1 Kyrle PA, Eichinger S. Deep vein thrombosis. Lancet 2005;365:1163-74

2 Heit JA, Silverstein MD, Mohr DN, Petterson TM, O’Fallon WM, Melton LI, 3rd. Predictors of survival after deep vein thrombosis and pulmonary embolism: a population-based, cohort study. Arch Intern Med 1999;159:445-53.

3 Prandoni P, Lensing AW, Cogo A, Cuppini S, Villalta S, Carta M, et al. The long-term clinical course of acute deep venous thrombosis. Ann Intern Med 1996;125:1-7.

4 Mohr DN, Silverstein MD, Heit JA, Petterson TM, O’Fallon WM, Melton LJ. The venous stasis syndrome after deep venous thrombosis or pulmonary embolism: a population-based study. Mayo Clin Proc 2000;75:1249-56.

5 Zornberg G, Jick H. Antipsychotic drug use and risk of first-time idiopathic venous thromboembolism: a case-control study. Lancet 2000;356:1219-23.

6 Parkin L, Skegg D, Herbison G, Paul C. Psychotropic drugs and fatal pulmonary embolism. Pharmacoepidemiol Drug Saf 2003;12:647-52.

7 Lacut K, Le Gal G, Couturaud F, Cornily G, Leroyer C, Mottier D, et al. Association between antipsychotic drugs, antidepressant drugs and venous thromboembolism: results from the EDITH case-control study. Fundam Clin Pharmacol 2007;21:643-50.

8 Ray J, Mamdani M, Yeo E. Antipsychotic and antidepressant drug use in the elderly and the risk of venous thromboembolism. J Thromb Haemost 2002;88:205-9.

9 Liperoti R, Pedone C, Lapane K, Mor V, Bernabei R, Gambassi G. Venous thrombo-embolism among elderly patients treated with atypical and conventional antipsychotic agents. Arch Intern Med 2005; $165: 2677-82$

10 Walker A, Lanza L, Arellano F, Rothman K. Mortality in current and former users of clozapine. Epidemiology 1997;8:671-7.

11 Hagg S, Spigset O. Antipsychotic-induced venous thromboembolism. CNS Drugs 2002;16:765-76.

12 Hammersley V, Hippisley-Cox J, Wilson A, Pringle M. A comparison of research general practices and their patients with other practicescross sectional survey in Trent. BrJ Gen Pract 2002;52:463-8.

13 Nazareth I, King M, Haines A, Ranger L, Myers S. Accuracy of diagnosis of psychosis in general practice computer system. $B M$ 1993;307:32-4

14 Lawrenson R, Todd JC, Leydon GM, Williams TJ, Farmer RD. Validation of the diagnosis of venous thrombo-embolism in general practice database studies. Br J Clin Pharmacol 2000;49:591-6.

15 Heit JA. The epidemiology of venous thrombo-embolism in the community: implications for prevention and management. J Thromb Thrombolysis 2006;21:23-9.

16 Motykie GD, Zebala LP, Caprini JA, Lee CE, Arcelus JI, Reyna JJ, et al. A guide to venous thrombo-embolism risk factor assessment. J Thromb Thrombolysis 2000;9:253-62.

17 Rosendaal FR, Van Hylckama Vlieg A, Tanis BC, Helmerhorst FM. Estrogens, progestogens and thrombosis. J Thromb Haemostasis 2003;1:1371-80.

18 Meier CR, Jick H. Tamoxifen and risk of idiopathic venous thromboembolism. Br J Clin Pharmacol 1998;45:608-12.

19 Royston P. Multiple imputation of missing values: update of ice. Stata J 2005; 5:527-36.

20 Jick H, Jick SS, Derby LE. Validation of information recorded on general practitioner based computerised data resource in the United Kingdom. BMJ 1991;302:766-8.

21 Majeed A. Sources, uses, strengths and limitations of data collected in primary care in England. Health Stat Q 2004;21:5-14.

22 jick H, Jick SS, Gurewich V, Myers MW, Vasilakis C. Risk of idiopathic cardiovascular death and nonfatal venous thrombo-embolism in 
women using oral contraceptives with differing progestagen components. Lancet 1995;346:1589-93.

23 Silverstein MD, Heit JA, Mohr DN, Petterson TM, O’Fallon WM, Melton LJ, 3rd. Trends in the incidence of deep vein thrombosis and pulmonary embolism: a 25-year population-based study. Arch Intern Med 1998;158:585-93.

24 Stolley P, Tonascia J, Tockman M, Sartwell P, Rutledge A, Jacobs M. Thrombosis with low-estrogen oral contraceptives. Am J Epidemiol 1975;102:197-208.

25 Ray W. Evaluating medication effects outside of clinical trials: newuser designs. Am J Epidemiol 2003;158:915-20.
26 Gill SS, Bronskill SE, Normand S-L, Anderson GM, Sykora K, Lam K, et al. Antipsychotic drug use and mortality in older adults with dementia. Ann Intern Med 2007;146:775-86.

27 Rochon PA, Normand S-L, Gomes T, Gill SS, Anderson GM, Melo M, et al. Antipsychotic therapy and short-term serious events in older adults with dementia. Arch Intern Med 2008;168:1090-6.

28 Douglas IJ, Smeeth L. Exposure to antipsychotics and risk of stroke: self controlled case series study. BMJ 2008;337:a1227.

29 Whitaker H, Farrington C, B. S, Musonda P. Tutorial in biostatistics: the self-controlled case series method. Stat Med 2006;25:1768-97.

Accepted: 22 June 2010

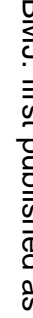

